\title{
Spectroscopy of the Three Planetary Nebulae in the Sagittarius Dwarf Galaxy
}

\author{
J. R. Walsh \\ ST-ECF, ESO, Karl-Schwarzschild Strasse 2 , D-85748 Garching, \\ Germany
}
A. A. Zijlstra
Department of Physics, UMIST, P.O. Box 88, Manchester, ¿K
D. Péquignot

Observatoire de Paris - Meudon, F-92195 Meudon Principal Cedex, France

Two planetary nebulae previously classified as Galactic were discovered, on the basis of their radial velocities, to be located in the Sagittarius Dwarf galaxy (Zijlstra \& Walsh, 1996, A\&A, 312, L21). At the distance of Sagittarius of $\sim 25 \mathrm{kpc}$, they are the closest extra-galactic PN. A detailed analysis based on ground-based spectra and radio continuum data was published (Dudziak et al. 2000, A\&A, 363, 717); it was shown that the two nebulae are on the same evolutionary track with initial mass of $1.2 \mathrm{M}_{\odot}$ and almost identical light element abundances. One of the nebulae, Wray 16-423, underwent PN ejection about $1500 y r$ before its twin He 2-436. On the basis of their similarity, a differential abundance analysis could be conducted. Third dredge-up carbon was found to be more abundant in $\mathrm{He} 2-436$ and the first conclusive evidence for thirddredge-up oxygen enrichment was revealed (Péquignot et al. 2000, A\&A, 361, L1).

A literature search for classified PN in the direction of the Sagittarius galaxy and its tidal tails, and with radial velocity incompatible with Milky Way Bulge membership, has been conducted. A new PN has been identified in Sagittarius - St-Wr 2-21 (PN G 005.2 -18.6), situated within $2^{\circ}$ of Wray 16-423. The radial velocity has been measured $\left(+133 \mathrm{kms}^{-1}\right.$ Heliocentric) and is within the errors of that of the mean of the two previously discovered Sagittarius PN. Low dispersion optical spectra of St-Wr 2-21 have also been obtained. The nebula is of higher ionization than He 2-436 and Wray 16-423 and is about $1^{\prime \prime}$ in diameter. A value of $12+\log (O / H)=8.59$ is derived, which is +0.25 dex higher than the mean value for the other Sagittarius PN. This higher abundance may reflect birth in a later star formation episode than for He 2-436 and Wray 16-423, or the typical dispersion in ISM abundance in a dwarf galaxy.

The triplet PN in Sagittarius and the known PN in the Fornax Dwarf galaxy, for which new deeper spectra have been obtained, are pillars in the study of dredge-up in lower metallicity stars and for investigations of the star formation and enrichment history of Local Group dwarf galaxies. 